\title{
Trajectory Tracking Control for Mobile Robot using Wavelet Network
}

\author{
Turki Y. Abdalla \\ Department of Computer Engineering \\ University of Basrah , Basrah,Iraq
}

\author{
Mustafa I. Hamzah \\ Department of Electrical Engineering \\ University of Basrah, Basrah,Iraq
}

\begin{abstract}
This paper present a new wavelet network control scheme for mobile robot path tracking. The particles swarm optimization (PSO) method is used for determining the optimal wavelet neural network parameters and the proportional integral derivative (PID) controller parameters, for the control of nonholonomic mobile robot that involves trajectory tracking using two optimized WNN controllers one for speed control and the other for azimuth control. The mobile robot is modeled in Simulink and PSO algorithm is implemented using MATLAB. Simulation results show good performance for the proposed control scheme.
\end{abstract}

\section{General Terms}

Mobile robot motion control system.

\section{Keywords}

Mobile Robot, Wavelet Neural Network, PSO, Trajectory Tracking .

\section{INTRODUCTION}

Robots are essential elements in society today. They are capable of performing many tasks repetitively and precisely without the help required by humans .A production line consisting of robots may shorten the time needed to make products and reduce the need for humans in industry. The word robot is used to refer to a wide variety of mechanical machines that are capable of movement. In order for a machine to be classified as a robot, it must possess artificial intelligence meaning that it must be at least partially controlled by a computer[1].

An autonomous mobile robots are being developed and used in many real world applications, for example, factory automation, underground mining, military surveillance, and even space exploration. In those applications, the mobile robots often work in unknown and inhospitable environments[2]. To survive, these robots must be able to constantly monitor and appropriately react to variation and uncertainty in their environments. A basic task that every autonomous mobile robot must perform is safely navigating itself from one point to another within its environment[3]. Two key ingredients that help the mobile robot to accomplish this navigation task are motion planning and control. The motion planning deals with the ability of a mobile robot to plan its own motion in its working space, and the motion control concerns with the ability of a mobile robot to follow or track a planned motion. Clearly, as mobile robot applications become more complex, the needs for more efficient and reliable motion planning and control are indispensable[4].
The aim of mobile robotic systems is the construction of autonomous systems that are capable of moving in real environment without the comforts of human operator by detecting objects by means of sensors or cameras and of processing this information into movement without a remote control.

\section{ANONHOLONOMIC MOBILE ROBOT MODEL}

Modeling of a differential drive mobile robot platform consists of kinematic and dynamic modeling. Kinematic modeling deals with the geometric relationships that govern the system and studies the mathematics of motion without considering the affecting forces. Dynamic modeling on the other hand is the study of the motion in which forces and energies are modeled and included. Each part of this system's modeling will be explained separately throughout this chapter.

The modeling of the kinematics of differentially steered wheeled mobile robots in a two dimensional plane can be done in one of two ways: either by Cartesian or by polar coordinates [5]. The modeling in Cartesian coordinates is the most routine and the kinematics will be limited to modeling in Cartesian coordinates.

\subsection{Kinematic Model of Mobile Robot}

Kinematics refers to the evolution of the position, and velocity of a mechanical system, without referring to its mass and inertia. The kinematic scheme of mobile robot consists of a platform driven by two driving wheels mounted on same axis with independent actuators and one free wheel (castor). The movement of mobile robot is done by changing the relative angular velocities of driving wheels. The assumptions are that the whole body of robot is rigid and motion occurs without sliding. Its wheel rotation is limited to one axis. Therefore, the navigation is controlled by the speed a change on either side of the robot [6]. This kind of robot has nonholonomic constraints. The kinematics scheme of a two wheel differential drive mobile robot is as shown in Figure(1), where $\{O, X, Y\}$ is the global coordinate, $v$ is the velocity of the robot centroid, $\omega$ is the angular velocity of the robot centroid, $v_{l}$ is the velocity of the left driving wheel, $v_{r}$ is the velocity of the right driving wheel, $2 b$ is the distance between two driving wheels, $r$ is the radius of each driving wheel, $x$ and $y$ are the position of the robot, and $\theta$ is the orientation of the robot. According to the motion principle of rigid body kinematics, the motion of a two wheel differential drive mobile robot can be described using equations (1) and (2), where $w_{l}$ and $w_{r}$ are angular velocities of the left and right driving wheels respectively[7]. 
$v_{l}=w_{l} r \quad, \quad v_{r}=w_{r} r$

The nonholonomic constraint equation of the robot is as following :

$$
\dot{x} \sin \theta-\dot{y} \cos \theta=0
$$

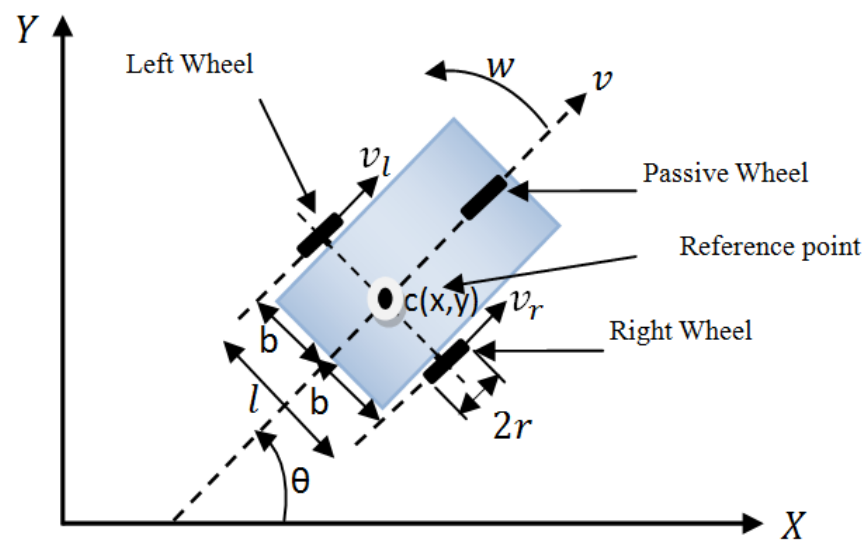

Figure(1): Kinematic Scheme of differential drive of the robot [5]

From (1) and (2) the following is obtained:

$v=\frac{\left(w_{r}+w_{l}\right)}{2} r, \quad w=\frac{\left(w_{r}-w_{l}\right)}{2 b} r$

Moreover, we can define the dynamic function of the robot as equation (5).

$\dot{x}=v \cos \quad, \dot{y}=\mathrm{v} \sin \theta \quad, \dot{\theta}=w$

Combining (4) and (5), we can obtain

$$
\left[\begin{array}{c}
\dot{x} \\
\dot{y} \\
\dot{\theta}
\end{array}\right]=\left[\begin{array}{cc}
\frac{r}{2} \cos \theta & \frac{r}{2} \cos \theta \\
\frac{r}{2} \sin \theta & \frac{r}{2} \sin \theta \\
-\frac{r}{2 b} & \frac{r}{2 b}
\end{array}\right]\left[\begin{array}{l}
w_{l} \\
w_{r}
\end{array}\right]
$$

to be more complex. Therefore, equation (6) should be decoupled. For $\theta$ is only related to $\omega, x$ and $y$ are only related to $v$. So, the kinematics model of the

$$
J q=\left[\begin{array}{c}
\dot{x} \\
\dot{y} \\
\dot{\theta}
\end{array}\right]=\left[\begin{array}{ll}
\cos \theta & 0 \\
\sin \theta & 0 \\
0 & 1
\end{array}\right]\left[\begin{array}{c}
v \\
w
\end{array}\right]
$$

where :

- $\quad \dot{x}$ and $\dot{y}:$ denote the velocity of the robot in the direction of $\mathrm{X}$-axis and $\mathrm{Y}$-axis, respectively.

\subsection{Dynamic Model of Mobile Robot}

The mobile robot considered here is shown in Figure(1). It consists of a vehicle with two driving wheels mounted on the same axis, and a front passive wheel for balance. The two driving wheels are controlled independently by motors.

Let the mobile robot be rigid moving on the plane. We assume that the absolute coordinate system OXY is fixed on the plane as shown in Figure 1. Then, the dynamic property of the robot is given by the following equation of motion :

Let the mobile robot be rigid moving on plane .We assume that the absolute coordinate system $\mathrm{O}-\mathrm{XY}$ is fixed on the plane as shown in Figure (1). then the dynamic property of the robot is given by the following equation of motion[9]

$\mathrm{M} \dot{v}=D_{r}+D_{l}$

$I_{v} \ddot{\varphi}=D_{r} l-D_{l} l$

$I_{w} \ddot{\varphi}_{i}+c \dot{\varphi}_{i}=\mathrm{k} u_{i}-\mathrm{r} D_{i} \quad \quad \mathrm{i}=\mathrm{r}, l$

Where each parameter and variable are defined by:
M : mass of robot.
$\varphi \quad$ : Azimuth of robot
$v \quad$ : Velocity of robot.
$I_{w} \quad$ : moment of inertia of wheel.
C : viscous friction factor
$\mathrm{K}$ : driving gain factor.
$\mathrm{r} \quad$ : radius of wheel.

- $\quad v$ : denote the linear velocity of the robot in the head direction of the robot.

- $\dot{\theta}=\omega$ : denotes the rotational velocity of the robot.

Finally, the kinematics model of the vehicle velocity $\boldsymbol{v}$ and the orientation $\boldsymbol{\theta}$ can be represented by the matrix as follows [10]

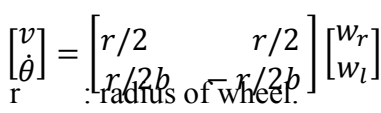

$\theta_{\mathbf{i}} \quad$ : rotational angle of wheel.

$u_{i} \quad$ : driving input

$I_{v} \quad$ : Moment of inertia around the C.G. of robot

$l \quad:$ Distance between left or right wheel and the C.G. of robot.

On the other hand, the geometric al relationships are among variables $\varphi, \mathrm{v}, \theta$

Given by:

$r \dot{\theta}_{r}=v+l \dot{\varphi}$

$r \dot{\theta}_{l}=v-l \dot{\varphi}$ 
From these equations, defining the state variable for the robot as $\mathrm{X}=[\varphi, v, \theta]$

, the manipulated variable as $\mathrm{u}=\left[\begin{array}{ll}u_{r} & u_{l}\end{array}\right]$ and the output variable as $y=[v, \theta]$ Yield as the following equation:

$\dot{\mathrm{x}}=\mathrm{Ax}+\mathrm{Bu}$

$\mathrm{y}=\mathrm{Cx}$

Where:

$\mathrm{A}=\left[\begin{array}{ccc}a_{1} & 0 & 0 \\ 0 & 0 & 1 \\ 0 & 0 & a_{2}\end{array}\right], \mathrm{B}=\left[\begin{array}{cc}b_{1} & b_{1} \\ 0 & 0 \\ b_{2} & -b_{2}\end{array}\right], \quad \mathrm{C}=\left[\begin{array}{lll}1 & 0 & 0 \\ 0 & 1 & 0\end{array}\right]$

$a_{1}=\frac{-2 \mathrm{c}}{\left(\mathrm{Mr}^{2}+2 I_{\mathrm{u}}\right)} \quad, \quad a_{2}=\frac{-2 \mathrm{cl}^{2}}{\left(I_{n} \mathrm{r}^{2}+2 I_{\mathrm{u}} I^{2}\right)}$

The equation (16) and (17) show the relation between the controller input torques $u r$ and $u l$ with $u v$ and $u \varphi$.

$u-1+11$
$u_{l}=u_{v}-u_{\varphi}$

velocity by using the measurement of the robot's velocity and $u_{\Phi}$ is the torque required for controlling the robot's azimuth by using the measurement of the robot's azimuth. In the simulations, we apply WNN controller, the computed torque controller used to control the path of the robot[9].

The velocity error $e_{v}$ and its rate, and the azimuth error $e_{\varphi}$ and its rate are considered as the inputs, and the driving torque required for controlling the two wheels $u_{r}$ and $u_{l}$ is considered as the output. Here, the input deviations $e_{v}$ and $e_{\varphi}$ are defined by:

$e_{v}=v_{d}-v$

$e_{\varphi}=\varphi_{d}-\varphi$

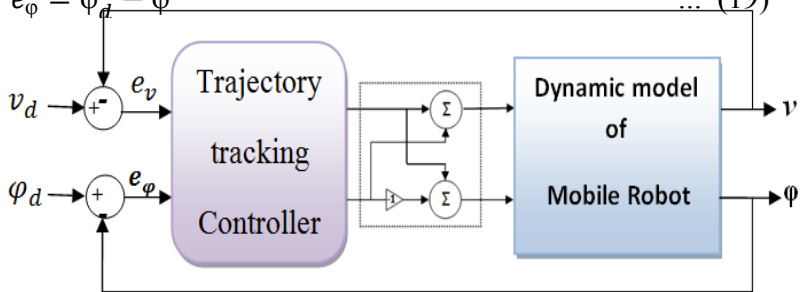

Figure (2): Block diagram of Trajectory control system

where $v_{d}, \varphi_{d}$ are the desire velocity and the desire azimuth, respectively. $v, \varphi$ are the actual velocity and the actual azimuth of the robot, respectively.

\section{PHYSICAL PARAMETERS:}

Physical parameters of the mobile robot are taken from [9] and shown in Table (1).

Table(1): Physical parameters of the mobile robot

\begin{tabular}{|c|c|c|}
\hline Parameter & Value & Unit \\
\hline $\mathrm{I} v$ & 10 & $\mathrm{Kg} \cdot \mathrm{m}^{2}$ \\
\hline $\mathrm{M}$ & 200 & $\mathrm{Kg}$ \\
\hline$l$ & 0.3 & $\mathrm{~m}$ \\
\hline $\mathrm{I} w$ & 0.005 & $\mathrm{Kg} \cdot \mathrm{m}^{2}$ \\
\hline $\mathrm{C}$ & 0.05 & $\mathrm{~kg} / \mathrm{s}$ \\
\hline $\mathrm{R}$ & 0.1 & $\mathrm{M}$ \\
\hline $\mathrm{K}$ & 5 & Unit less \\
\hline
\end{tabular}

\section{WAVELET NEURAL NETWORK}

The proposed wavelet neural network shown in Figure(3) which represent the classified model of multi-input and multioutput (MIMO) WNN with three layers. The node number of the input layer is $\mathrm{M}$, the hidden layer is $\mathrm{K}$ and the output layer is $\mathrm{N}$. The impulse function of hidden layer is wavelet basis function.

The impulse function of output layer is sigmoid function. The formula for the computation is as follows[10].

$\sigma(u)=\frac{1}{1+e^{-u}}$

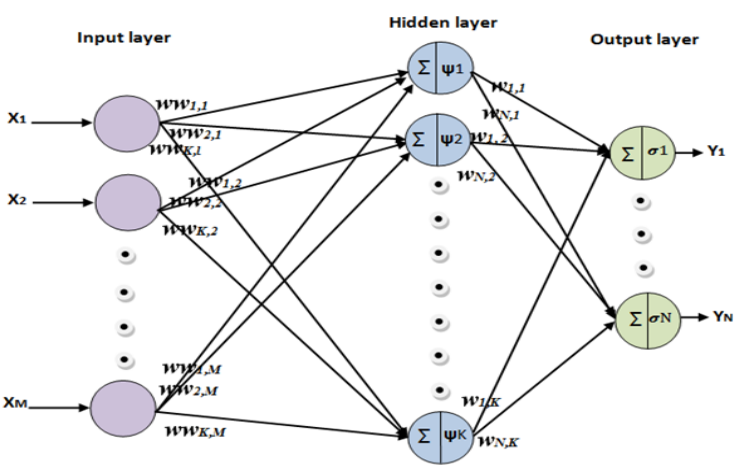

Figure (3): The structure diagram of WNN

If the training sample set is $X=\left[X_{1}, X_{2}, \ldots \ldots, X_{M}\right]$, the corresponding actual output is $y=\left[y_{1}, y_{2} \ldots \ldots y_{N}\right]$, the expected output is $\mathrm{D}=\left[\mathrm{D}_{1}, \mathrm{D}_{2}, \ldots \ldots, \mathrm{D}_{\mathrm{N}}\right]$, the input sample size is $\mathrm{N}$, the sum of the output layer deviations is $\mathrm{E}(\mathrm{N})$,

$$
\begin{aligned}
& Y_{i}=\sigma\left(u_{i}\right)=\sigma\left[\sum_{k=1}^{K} W_{i, k} \psi_{a k, b k}\left(\sum_{m=1}^{M} w w_{k, m} x_{m}\right)\right] \\
& i=1,2,3, \ldots \ldots \ldots \ldots N \\
& E(N)=\frac{1}{N} \sum_{i=1}^{N}\left(D_{i}-Y_{i}\right)^{2}
\end{aligned}
$$


$\mathrm{N}$ is the training samples number, $D_{i}$ is the ideal output value of the output node $i, Y_{i}$ is the actual output value of the output node $\mathrm{i}$.Wavelet network in Figure(3) shows, after the number of input layer, hidden layer and output layer are make certain, the key to construct a suitable wavelet network is to decide the parameters $\left\{w w_{m, k}, a_{k}, b_{k} k, w_{k, n}\right\}$. And the parameters can be confined by the optimization of error function $\mathrm{E}(\mathrm{N})$ [11]. the wavelet network operation consist of two phases. in the first phase, the network architecture is determined for certain application. In the second phase the parameter of the network are updated so that the approximation errors are minimized two type of training algorithms used gradient and PSO algorithm.

\section{WAVELET NEURAL NETWORK BASED ON PSO}

WNN-PSO use the particles search the link weights, threshold between each layer in their searching space, and telescopic translation parameter $a_{k}$ and $b_{k}$ in the wavelet basis function. In the particles searching procession, its iterative formula is simple, and its calculation speed is faster than the gradient descent method. It can jump out of the local minimum based on the adjustment of the inertia weight function easily[12].

Establish the wavelet neural networks according to the Figure(3). The element of the location vector $x$ in the Particle Swarms is defined as the link weights and telescopic translation parameter $a_{k}$ and $b_{k}$ between each layer of the wavelet neural networks. Fitness function is the square error function $\mathrm{E}(\mathrm{N})$ of the wavelet neural networks. As seen in the equation (26).

\section{WNN-PSO TRAINING ALGORITHM PROCESS:}

(1) Set the of the initial value telescopic factor $a_{k}$ and the translation factor $b_{k}$ of the wavelet networks parameters according to the method of reference.[13]

(2) Initiate the parameters of the Particle Swarms: Set the particle number $\mathrm{m}$; fitness threshold $\varepsilon$; set the maximum allowable number of iterative step MaxIter ; set the accelerating factor $c_{1}, c_{2}$; set the minimum min $\omega$ and maximum $\max w_{\max }$ of $\omega$; the particle location $\mathrm{X}$; speed $\mathrm{V}$ initial to the random number between 0 and 1 .

(3) Iterative update the location vector $x$ and the speed $v$ of every particle according to the formula of the Particle Swarm Optimization (23),(24) and (25),

$$
\begin{aligned}
& v_{i d}=w v_{i d}+c_{1} r_{1}\left(p_{i d}-x_{i d}\right)+c_{\ni} r_{\supset}\left(p_{\text {rd }}-x_{i d}\right) \\
& x_{i d}=x_{i d}+v_{i d} \\
& w=w_{\text {max }}-\frac{\text { Iter }}{\text { MaxIter }}\left(w_{\text {max }}-w_{\text {min }}\right)
\end{aligned}
$$

record the history optimal location vector $P_{i d}$ of every particle and overall optimal location vector $P_{g d}$. Calculate the fitness value according to formula (26), record the fitness value Fitness $P_{i d}$ and Fitness $P_{g d}$ corresponding to $P_{i d}$ and $P_{g d}$.

$\mathrm{E}=\frac{1}{\mathrm{~N}} \sum_{\mathrm{p}=1}^{\mathrm{N}}[\mathrm{e}(\mathrm{p})]^{2} \quad$ and $\quad \mathrm{e}(\mathrm{p})=\mathrm{y}^{\mathrm{d}}-\mathrm{y}(\mathrm{p})$

where $y(p)$ is the model output, and $y d(p)$ is the desired output for a given $p t h$ input pattern. $\mathrm{N}$ represents the total training patterns.
(4) Judge if the fitness value reach to the setting value, and if the iterations number reach to the highest. If the Fitness $P_{g d} \leq$ setting value or the iterations number reach to the highest, the iterations over, or transferred to step (3).

(5) Let $g d P$ into every weights, telescopic vector $\vec{a}$ and translation factor $b$ of the wavelet neural network, calculate the networks output. Figure(4) shows the Mobile robot optimization network model based on the Particle Swarm Optimization PID controller used to improve response and reduce learning cycle.

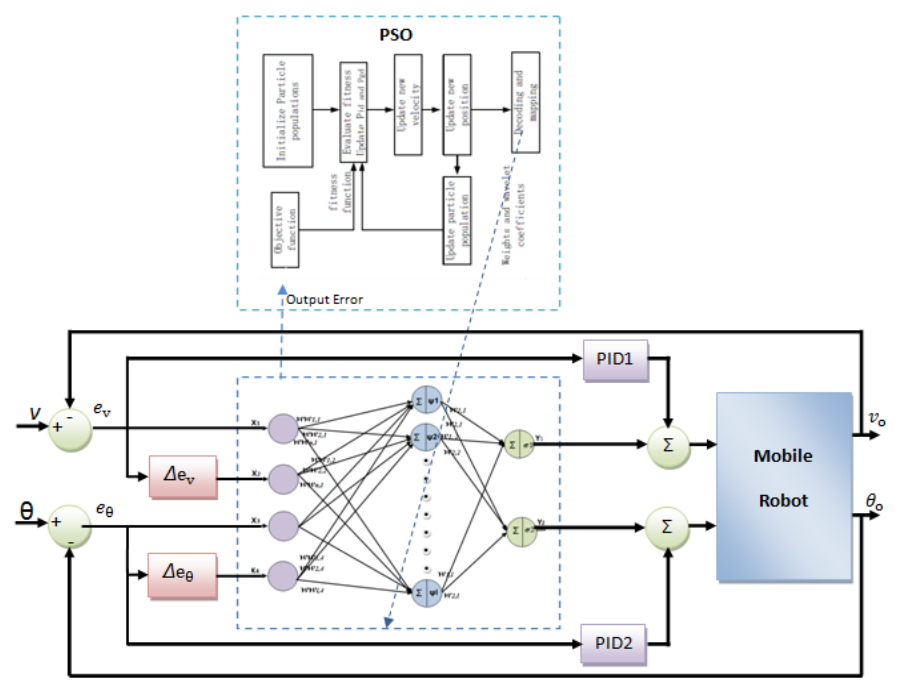

Figure( 4 ) :Mobile Robot Control system diagram using PSO-WNN

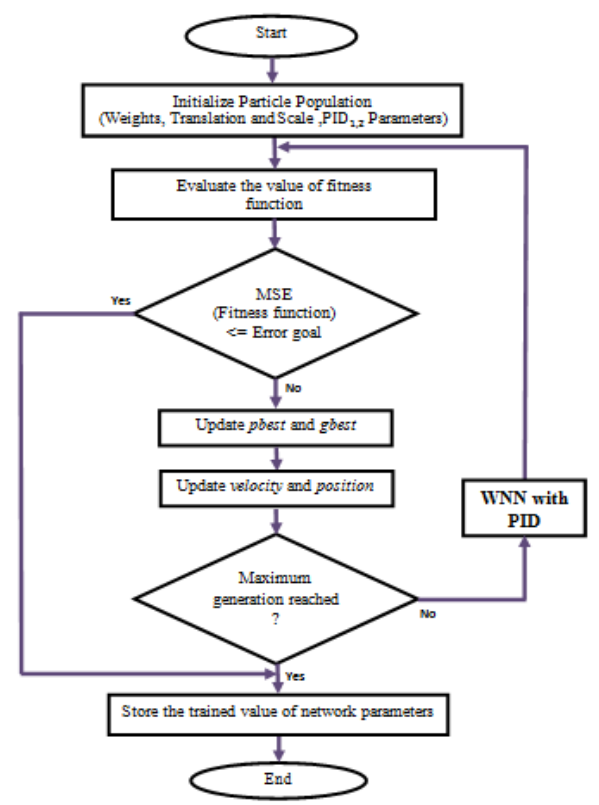

Figure(5 ): PSO aided WNN Training Algorithm

\section{SIMULATION RESULTS:}

To simulate the above mobile robot model, Matlab simulation Program ver. 2008 and use the $4^{\text {th }}$ order Runge-Kutta-Gill method for simulink setting .It is also assumed that the control sampling period is $10 \mathrm{~ms}$.In addition, the reference velocity 
$v_{d}$ is given as $0.25 \mathrm{~m} / \mathrm{s}$ and the initial value of state variable is given as $X_{0}=\left[\begin{array}{lll}0 & 0 & 0\end{array}\right]$ for the state space parameter calculations tuned PID error constants values by using PSO optimization method. The proposed scheme in Figure(4) shows trajectory tracking mobile robot control system using one MIMO wavelet neural network consist of three layers input layer of four neurons, hidden layer (mother wavelet layer) of fifty neurons and output layer of two neurons one output used for linear velocity control and the other output used for azimuth control the whights, translation and dilation factors for WNN and two PID s tuned parameters are selected depending on PSO optimization method.

Table(2) shows the initilization parameters for PSO operation. which use Mexican hat wavelet in the hidden layer. Table( 3 ) shows the tuned parameters using PSO.

\section{Table(2): PSO initialization parameters Mobile Robot} system

\begin{tabular}{|c|c|}
\hline PSO_Parameters & Value \\
\hline Size of the swarm " no of birds " & 50 \\
\hline Maximum iteration number & 200 \\
\hline Dimension & 408 \\
\hline PSO parameter C1 & 2 \\
\hline PSO parameter C2 & 2 \\
\hline Wmax & 0.9 \\
\hline Wmin & 0.3 \\
\hline
\end{tabular}

Table(2): Tuned parameters using PSO

\begin{tabular}{|c|c|c|c|c|c|c|c|c|}
\hline Err Cons. & $K$ & & $K$ & $K$ & $K$ & & & \\
\hline Values & 38 & 246 & 0 & 8.5 & 63 & 0.11 & 0.65 & 144.2 \\
\hline
\end{tabular}

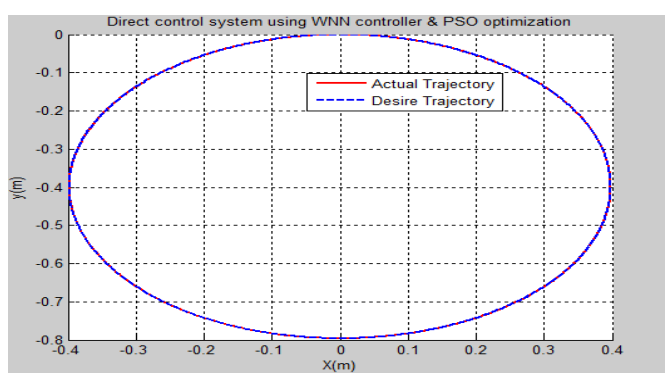

Figure (6 ): Circle Trajectory Tracking

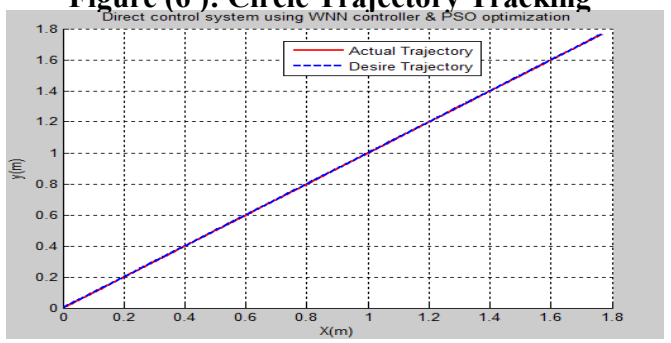

Figure (7 ): Linear Trajectory Tracking

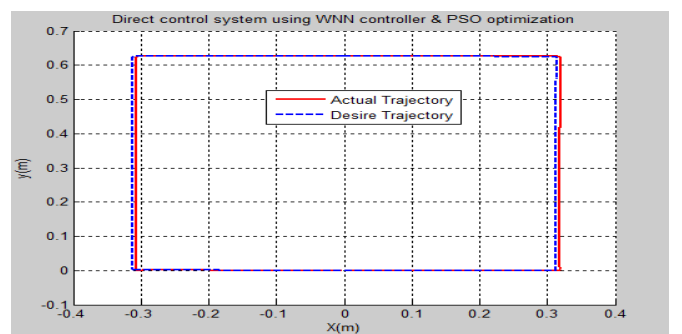

Figure (8 ): Square Trajectory Tracking
Table(4) shows MSE values for different trajectories

Table(4):MSE values for different trajectories

\begin{tabular}{|c|c|c|c|}
\hline Trajectory & Circle & Linear & Square \\
\hline MSE & $2.2097 \mathrm{e}-006$ & $2.7932 \mathrm{e}-004$ & 0.0073 \\
\hline
\end{tabular}

\section{CONCLUSION}

Wavelet control scheme is built and implemented in matlab / simulink software package and it is succeeded to solve the trajectory tracking problem of mobile robot. A tracking control problem for the speed and azimuth of a mobile robot driven by two independent wheels has been solved by using Mexican hat Wavelet Neural Network controller optimized by using PSO algorithm. The Particle Swarm Optimization method is utilized to tune the parameters and weights of WNN . It gives good results in short time relatively with other optimization methods. The effectiveness of the proposed method was illustrated by performing the simulation for circular, linear and square trajectory. Simulation results show good tracking performance with small Mean square error.

\section{REFERENCES}

[1] Hachour O.," Novel Mobile Robot Path planning Algorithm ", international journal of system applications, engineering and development Issue 4, Volume 4, 2010.

[2] Peter S. and Anna J.," Tracking trajectory of the mobile robot Khepera II using approaches of artificial intelligence ", Acta Electrotechnica et Informatica, Vol. 11, No. 1, 2011.

[3] Hachour O. "Path planning of Autonomous Mobile robot",international journal of system applications, engineering and development Issue 4, Volume 2, 2008.

[4]Pornporm B.,"Online path replanning of autonomous mobile robot with Spline based Pornporm Boonporm algorithm", International Conference on System Modeling and Optimization, vol. 23,2002.

[5] Dongkyoung Chwa ," Sliding-Mode Tracking Control of Nonholonomic Wheeled Mobile Robots in Polar Coordinates ",IEEE TRANSACTIONS ON CONTROL SYSTEMS TECHNOLOGY, VOL. 12, NO. 4, JULY 2004.

[6]Wheekuk Kim, Seung-Eun Lee and Byung-Ju Yi,"Mobility Analysis of Planar Mobile Robots",IEEE, International conference on robotics and automation,pp. 28612867,2002

[7] Farhan A. Salem, "Dynamic and Kinematic Models and Control for Differential Drive Mobile Robots", International Journal of Current Engineering and Technology ,ISSN 2277 - 4106, Vol.3, No.2 , 2013.

[8]Peng J., Wang Y. and Sun W., "Trajectory-Tracking Control for Mobile Robot Using Recurrent Fuzzy Cerebellar Model Articulation Controller", Neural Information Processing - Letters and Reviews, Vol. 11, No. 1, January 2007.

[9] Watanabe K., Tang J., Nakamura M., Koga S. ,and Fukuda T., "Mobile Robot Control Using Fuzzy-Gaussian Neural 
Networks", IEEE/RSJ International Conf. on Robots and system, pp. 919-925, 1993.

[10] Prasanta K. Pany," Short-Term Load Forecasting using PSO Based Local Linear Wavelet Neural Network",International Journal of Instrumentation, Control and Automation (IJICA) ISSN : 2231-1890 Vol.1, No.2, 2011.

[11] Yunlong H., Shiming Y., "Explosive Ordnance Disposal Robot Path Planning Based on Danger Model Immune
Wavelet Neural Network",Advances in information Sciences and Service Sciences, Vol.3, No.11, 2011.

[12] Mao H., Pan H., "Wavelet neural network based on particle swarm optimization algorithm and its application in fault diagnosis of gearbox ", Journal of vibration and shock,vol. 26,no. 5, pp. 133-136,2007.

[13] Pan H., Huang J., and Mao H., "Research of Faultcharacteristic Extractive technology Based on Particle Swarm Optimization", IEEE, pp.1941-1946, 2009. 